Programs of the Federal Motor Carrier Safety Administration (FMCSA)

encompass a range of issues and disciplines, related to motor carrier safety and security. FMCSA's Office of Analysis, Research and Technology defines a "research program" as any systematic study directed toward fuller scientific discovery, knowledge, or understanding that will improve safety, and reduce the number and severity of commercial motor vehicle crashes. Similarly, a "technology program" is a program that adopts, develops, tests, and/or deploys innovative driver and/or vehicle best safety practices and technologies that will improve safety and reduce the number and severity of commercial motor vehicle crashes. An "analysis program" is defined as economic and environmental analyses done for agency rulemakings, as well as program effectiveness studies, state-reported data quality initiatives, and special crash and other motor carrier safety performance-related analyses. A "large truck" is any truck with a Gross Vehicle Weight rating or Gross Combination Weight rating of 10,001 pounds or greater.

Currently, the FMCSA Office of Analysis, Research and Technology is conducting programs in order to produce safer drivers, improve safety of commercial motor vehicles, produce safer carriers, advance safety through information-based initiatives, and improve security through safety initiatives. The study described in this Tech Brief was designed and developed to support the strategic objective to produce safer drivers. The primary goals of this initiative are to ensure that commercial drivers are physically qualified, trained to perform safely, and mentally alert.

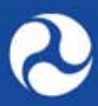

U.S. Department of Transportation

Federal Motor Carrier Safety Administration Office of Analysis, Research and

Technology

1200 New Jersey Ave. SE

Washington, DC 20590

\section{Evaluating the Safety Benefits of a Low-Cost Driving Behavior Management System in Commercial Vehicle Operations}

Motor vehicle crashes are often predictable and preventable. Yet, many drivers choose to behave in ways that put themselves and others at risk for a vehicle crash and/or serious injuries. Behavioral safety programs are advantageous because they are easy to implement, easy to teach, and may be implemented successfully in the setting where the problem occurs to increase safety-related work behaviors. Almost all prior behavioral safety research has been applied in work settings where employees can systematically observe the safe versus atrisk behaviors of their coworkers. In contrast, commercial truck and bus drivers typically work alone and in relative isolation and thus require alternative strategies. Until recently, the primary problem with implementing behaviorbased programs has been getting quality behavioral data on driving behaviors. If behavioral approaches can be integrated with technologies that monitor driver behavior, fleet safety managers would have an effective tool to improve safetyrelated behaviors.

New technologies are available that provide objective measures of driver behavior. These in-vehicle technologies are able to provide continuous measures on a wide variety of driving behaviors previously unavailable to fleet safety managers. Some driving behavior monitoring systems (DBMSs) use in-vehicle video technology to record driver behavior. These recordings can be used by fleet safety managers to provide feedback on safe and at-risk driving behaviors to reduce crash and injury risk. Thus, DBMSs have the potential to be used in conjunction with behavioral safety techniques to reduce a variety of at-risk behaviors. The current study provides an independent evaluation of a commercially available low-cost DBMS with commercial drivers.

\section{Research Design}

This quasi-experiment used a simple $\mathrm{A}^{4} \mathrm{~B} 13$ design; where " $\mathrm{A}$ " and " $\mathrm{B}$ " refer to the baseline and intervention phases, respectively. The superscript refers to the number of weeks in each phase (i.e., "4" refers to 4 weeks). During the 4-week baseline phase, drivers drove an instrumented vehicle during their normal, revenue-producing deliveries. The event recorder was configured to record safety-related events as normal; however, the feedback light was disabled (under normal operation, a light on the event recorder flashed each time an event was recorded) and no driver counseling occurred. Immediately following the 4-week baseline, the 13-week intervention phase began, during which drivers drove an instrumented vehicle during their normal, revenue-producing deliveries. During this time, the event recorder recorded safety-related events as normal and the "coaching" program was enabled (i.e., the feedback light was activated and safety managers followed the recommended coaching protocol).

\section{Participants and Setting}

Carrier A was a long-haul carrier located in the Southeastern United States that primarily delivered dry goods. A total of 50 drivers had an event recorder 
installed in their trucks (36 drivers completed data collection). A total of 46 drivers at Carrier A signed an informed consent form that allowed researchers to send questionnaires to participating drivers. The mean age of these 46 drivers was 44 years old (range $=23$ to 61 years old).

Carrier B was a local/short-haul carrier located in the Northwestern United States that primarily delivered beverage and paper goods. A total of 50 drivers had an event recorder installed in their trucks (41 drivers completed data collection). A total of 30 drivers at Carrier B signed an informed consent form that allowed researchers to send questionnaires to participating drivers. The mean age of these 30 drivers was 50 years old (range $=27$ to 71 years old).

\section{Data Collection Process}

A product vendor, DriveCam, was responsible for all data collection. The event recorder captured two camera views: (1) driver's face view, and (2) forward-facing view. The event recorder had three accelerometers ( $\mathrm{y}-, \mathrm{x}-$, and $\mathrm{z}$-axis) that triggered an event to be recorded. If the criterion was met or surpassed (e.g., greater than or equal to $|0.5 \mathrm{~g}|$ ), the event recorder saved 12 seconds of video (i.e., 8 seconds prior to the criterion being met or surpassed and 4 seconds after). The video and quantitative data were automatically sent to the vendor's headquarters in San Diego, CA, via cellular transmission. Once received the data were reviewed, reduced (i.e., data analysts marked the presence of specific variables pertaining to the event), and uploaded to a server. While all safety-related events were uploaded to the server for review, only those safety-related events that exceeded a certain threshold (or "Event Score") were requested to be reviewed with the driver. Event Scores in the current study ranged from 0 to 11. Typically, an Event Score greater than or equal to 5 was marked to be reviewed by the safety manager with the driver present; however, it was ultimately up to the safety manager which safety-related events were reviewed with the driver. Once on the server, the authors and safety managers, only during the intervention phase had access to the data via proprietary software (which was accessible via the Internet). Researchers checked the software each day and recorded the frequency of safety-related events, severity, driving behaviors, date, driver\#, and quantitative data.

\section{Results}

Hypothesis 1 - There will be a significant reduction in the mean rate of safety-related events from baseline to intervention

Carrier A - During the 4-week baseline phase, 58 safety-related events were captured by event recorders ( 2 collisions and 56 risky driving events) from the 36 drivers who completed the study. These drivers drove a total of 291,869 miles during the baseline phase. A rate was calculated to account for exposure (i.e., frequency of safety-related events/10,000 vehicle miles traveled [VMT]). The mean rate of safety-related events/10,000 VMT during the baseline phase was 1.9 safety-related events.

During the 13-week intervention phase, 141 safety-related events were captured by event recorders ( 2 collisions and 139 risky driving events) over 1,170,721 miles. The mean rate of safety-related events/10,000 VMT during the intervention phase was 1.2 safety-related events. A paired sample $t$ test found the mean rate of safety-related events/10,000 VMT during the intervention phase (1.2 safety-related events/10,000 VMT) was significantly lower than the mean rate of safety-related events/10,000 VMT during the baseline phase (1.9 safety-related events/10,000 VMT; $t_{(35)}=1.7$, $p=0.046$ ). Thus, the 38.1 percent reduction in the mean rate of safety-related events/10,000 VMT from the baseline to intervention phases was significant.

Carrier B - During the 4-week intervention phase, 65 safety-related events were captured by the event recorder ( 1 collision and 64 risky driving events) from the 41 drivers who completed the study. These 41 drivers drove a total of 162,492 miles during the baseline phase. As indicated above, a mean rate of safety-related events/10,000 VMT was calculated to account for exposure. 
The mean rate of safety-related events/10,000 VMT during the baseline phase was 4.02.

During the 13-week intervention phase, a total of 117 valid safety-related events were captured by the event recorder ( 2 collisions and 115 risky driving events). These same 41 drivers drove a total of 615,403 miles. The mean rate of safety-related events/10,000 VMT during the intervention phase was 1.93. A paired sample $t$ test found that the mean rate of safety-related events/10,000 VMT during the Intervention phase (1.9 safety-related events/10,000 VMT) was significantly lower than the mean rate of safety-related events/10,000 VMT during the baseline phase (4.0 safety-related events/10,000 VMT; $\left.t_{(40)}=1.88, p=0.03\right)$. Thus, the 52.2 percent reduction in the mean rate of safety-related events/10,000 VMT from the baseline to intervention phases was significant.

Additional analyses are presented in the main body of the current report. These include examination of a second hypothesis (to wit: there will be a significant reduction in the mean rate of severe safety-related events from baseline to intervention, which was not supported at Carrier A or Carrier B), analyses of questionnaire data, severe safety-related events, and post-hoc analyses of drivers who did and did not receive feedback.

\section{Conclusions}

In interpreting these results, two issues are noteworthy. First, it appears Carrier B had more significant decreases than Carrier A in the mean rate of safety-related events/10,000 VMT (based on percentage reduction); however, concluding differential intervention impact is risky because Carrier A drove more safely than Carrier B during the baseline phase (1.9 versus 4.0 safety-related events/10,000 VMT). For example, Carriers A and B likely experienced different safety-related environmental conditions due to the predominant roads driven. Long-haul drivers typically drive on rural divided roads (e.g., highways), while local/short-haul drivers typically drive on urban undivided roads. Nonetheless, both carriers had substantial safety improvements from the DBMS.

Second, drivers were aware the instrumented vehicles were recording their driving behaviors; thus, it is possible that drivers altered their performance accordingly (i.e., subject reactivity). However, it is unlikely this awareness influenced intervention impact as any reactivity to the event recorders or the DBMS was constant across both phases, and any effect of reactivity to being observed is likely to be most prominent at the beginning of such procedures. In fact, the data obtained during the baseline phase may have been understated, resulting in a less robust effect during the intervention phase. If this was the case, note that event recorders were installed in vehicles at Carriers A and B several weeks prior to the start of data collection. Thus, drivers would have become familiar with the presence of the event recorders by the time data collection began. As such, it is unlikely the results were impacted by reactivity effects since the strong, positive benefits of the DBMS in reducing safety-related events in this study were robust.

\section{Recommendations}

The goal of the current study was to assess the efficacy of a commercially available low-cost DBMS in an applied setting while normal, revenue-producing deliveries were made. Thus, no attempt was made to deviate significantly from the existing DBMS. As prior research has found the combination of goal setting and feedback to be the optimal approach, future studies assessing the efficacy of a DBMS should consider the addition of goal setting training and directly assessing participants' goals. The current study did not assess implicit goal setting; thus, variations in goal setting among drivers could have been the reason for differential behavior change among drivers. The current DBMS was successful in significantly reducing the mean rate of safety-related events/10,000 VMT (by 37 and 52.2 percent at Carriers A and B, respectively).

Though the safety benefits identified in this study were significant, it is possible that carriers may be reluctant to adopt such programs without a compelling case for return-on-investment. That is, although improved safety is a key outcome of the DBMS used in this study, it may not be sufficient 
Full report title:

Evaluating the Safety Benefits of a Low-Cost Driving Behavior

Management System in

Commercial Vehicle Operations

(Doc. No. FMCSA-RRR-10-033)

Performed by:

Center for Truck and Bus Safety

Virginia Tech Transportation

Institute

3500 Transportation Research

Plaza (0536)

Blacksburg, VA 24061

Authored by:

Jeffrey S. Hickman and

Richard J. Hanowski

Key Words:

behavioral safety, carrier safety,

CMV ,commercial motor vehicle,

driver behavior, low-cost driving

behavior management system,

onboard safety monitoring, truck

Notice:

Tech Briefs are disseminated under

the sponsorship of the U.S.

Department of Transportation

(USDOT) in the interest of

information exchange. Tech Briefs

provide a synopsis of the study's

final publication. Tech Briefs do not

establish policies or regulations, nor

do they imply USDOT endorsement

of the conclusions or

recommendations. The U.S.

Government assumes no liability

for its contents or their use.

\section{Web Site:}

All FMCSA Tech and Analysis

Briefs may be accessed at

www.fmcsa.dot.gov. to evoke widespread adoption of the technology.

As such, the authors recommend that a follow-on cost-benefit analysis research be directed at assessing the return-on-investment of a DBMS. The authors recommend that such an assessment include the costs associated with implementing and maintaining the DBMS program as well as the direct (e.g., damage, health care, etc.) and indirect (e.g., legal fees, insurance costs, etc.) costs associated with reduced crashes and violations. If it can be shown that there a significant safety benefit from a DBMS and associated cost savings to carriers due to the associated reduction in safety events, then a strong case may be made for the efficacy of a DBMS program. 